\title{
Suicidal Adolescents' Social Support from Family and Peers: Gender-Specific Associations with Psychopathology
}

\author{
David C. R. Kerr, ${ }^{1}$ Lesli J. Preuss, ${ }^{2}$ and Cheryl A. King ${ }^{1,3,4}$
}

Received July 30, 2003; revision received February 10, 2005; accepted May 6, 2005

\begin{abstract}
Perceptions of social support from family, non-family adults, and peers were examined in relation to the psychopathology reported by 220 suicidal adolescents ( 152 females) during a psychiatric hospitalization. Results of regression analyses showed that, among females, family support was negatively related to hopelessness, depressive symptoms, and suicidal ideation. Among males, peer support was positively associated with depressive symptoms and suicidal ideation. Across gender, more peer support was associated with more externalizing behavior problems; whereas, family support was negatively related to these problems and to alcohol/substance abuse. Paralleling normative findings, age was positively associated with peer support, and females perceived more peer support than did males. Findings extend previous research on social support to suicidal adolescents, and broaden the literature by examining extrafamilial support and a broader range of relevant psychopathology. That is, perceived social support relates to psychiatric impairment differentially by gender, and normative, age-related variations in perceptions of social support are detected even among highly impaired adolescents. Clinical implications and directions for future research are discussed.
\end{abstract}

KEY WORDS: suicide; social support; adolescence; depression; gender differences.

Suicide is the third leading cause of death among adolescents in the United States (Anderson, 2002). Although completed suicide is rare even among adolescents who attempt suicide or report serious suicidal ideation, these youth most often experience severe psychopathology and functional impairment (reviewed in King, 1997). Additionally, suicidal adolescents are at risk for continued suicidal behavior and psychiatric illness during adulthood (Goldston, Daniel, \& Reboussin, 1999). Unfortunately, few randomized controlled intervention trials (RCTs) have been conducted with suicidal adolescents. To date, only Huey et al. (2004) and Wood, Trainor, Rothwell, Moore, and Harrington (2001) have found signifi-

\footnotetext{
${ }^{1}$ Department of Psychiatry, University of Michigan, Ann Arbor, Michigan.

2 New York State Psychiatric Institute, New York, New York.

${ }^{3}$ Departments of Psychology, University of Michigan, Ann Arbor, Michigan.

${ }^{4}$ Address all correspondence to Cheryl King, Department of Psychiatry, Child \& Adolescent Division, University of Michigan Health System, 1500 E. Medical Center Drive, Ann Arbor, Michigan 48109-0295; email: kingca@med.umich.edu.
}

cant intervention effects on suicide attempts or repeated deliberate self-harm, and no RCTs have demonstrated reductions in suicidal ideation, except in subgroup analyses (e.g. Harrington et al., 1998; King et al., in press). Of further concern, though some interventions have been shown to lessen psychopathology associated with suicidality, suicidal adolescents frequently do not adhere to recommended treatments (Spirito, Boergers, \& Donaldson, 2000). Thus, it should be a priority to make use of all available resources to intervene with suicidal adolescents and enhance treatment adherence.

The social support that suicidal adolescents receive from family members and their broader networks represents one potential resource in their treatment. Social support frequently acts as a buffer against stress for adolescents (e.g. Wills \& Cleary, 1996). Such support may be particularly critical for suicidal adolescents, who often have experienced more traumatic events and life stress (de Wilde, Kienhorst, Diekstra, \& Wolters, 1992). At the same time, however, suicidal adolescents tend to perceive their families as less engaged, affectionate, and confiding than either non-clinical adolescents or non-suicidal, 
depressed adolescents (King, Segal, Naylor, \& Evans, 1993). Thus, multiple stressors may impact these vulnerable adolescents, without the mitigating effects of an emotionally supportive social network. Although enhancement of social support has been targeted as an intervention in a recent treatment study with suicidal adolescents (King et al., in press), relatively little is known about these youth's actual experience of support from their social networks.

\section{Sources of Suicidal Adolescents' Social Support}

Previous studies of the relationship between social support and adolescent maladjustment generally have focused on specific sources of support, especially family support, or have studied community or outpatient adolescent samples. No studies have considered the relative and unique importance of family, non-family adult, and peer support to psychopathology in suicidal youth.

Studies suggest that inadequate family support is most deleterious to adolescent functioning. For example, the negative appraisals that outpatient adolescents make about unsupportive family environments appear to be more strongly related to depression than their subjective evaluations of other relationships (Cumsille \& Epstein, 1994). Research also has consistently demonstrated associations between unsupportive family environments and suicidal behavior in adolescents. For example, suicidal adolescent inpatients perceive lower levels of family support than non-suicidal inpatients or non-patient controls (King et al., 1993), and poor family support predicts adolescents' suicidal behavior and ideation in the six months following psychiatric hospitalization (King et al., 1995). In depressed adolescent samples, low support continues to predict suicidality into adulthood (Lewinsohn, Rohde, Seeley, \& Baldwin, 2001). Low family support not only is linked contemporaneously and prospectively with suicidal behavior, it is associated with poor treatment adherence. For example, King, Hovey, Brand, Wilson, and Ghaziuddin (1997) found that a less involved and less affectionate father-adolescent relationship was one predictor of poor treatment adherence for adolescent inpatients. Thus, the social support provided by or missing from families has significant implications for suicidal adolescents' functioning and treatment, and must be considered before examining the contributions of additional support sources.

Though infrequently studied in relation to adolescents' impairment, non-family adults, such as teachers, family friends, and friends' parents, may offer additional or compensatory social support to some youth. For example, in their community sample, Slavin and Rainer (1990) found that, among adolescent girls, higher social support from non-family adults predicted fewer symptoms of depression after six months, even after controlling for initial symptoms and other sources of support. The importance of these types of relationships in the lives of suicidal adolescents has not been studied, even though the support available from non-family adults is a promising resource in the treatment of these youth (King et al., in press).

How peer support relates to impairment among suicidal adolescents also needs to be better understood. Whether support from peers supplements, interacts with, or overlaps with other sources of support, appears to depend on the population being studied. For example, in community samples, research suggests friendships may compensate for effects of weak family support on adolescents' adjustment (Gauze, Bukowski, Aquan-Assee, \& Sippola, 1996). Notably, Slavin and Rainer (1990) found that higher peer support predicted fewer symptoms of depression after six months in a community sample of female adolescents, even after controlling for family and non-family adult support. In more impaired populations, however, peer support may not be able to make up for low family support, as suggested by Cumsille and Epstein (1994). In their study of outpatient adolescents, these authors found that peer support was not related to depression after family support was controlled; greater peer support was related to less depression only when support from the family environment was relatively high.

Research has suggested that, in some cases, peer support is associated with greater dysfunction for adolescents. Indeed, the dubious nature of peer influence in clinical populations has been explored in the literature on "deviancy training" among male adolescents with conduct disturbances. That is, for some vulnerable youth, interactions with deviant peers cause increases in antisocial behavior, such as substance use, delinquency, and violence (Dishion \& Owen, 2002; Dishion, Spracken, Andrews, \& Patterson, 1996). Since what some youth perceive as support from peers may actually include shared risk-taking and reinforcement of antisocial behavior and maladaptive emotional coping, the potentially negative effects of peer "support" deserves further study within suicidal adolescent populations. For example, in a community sample, adolescents' suicidal behavior was associated with suicidal behaviors in their friends (Prinstein, Boergers, \& Spirito, 2001). This effect was magnified with increased adolescent depression and family dysfunction, two risk factors of considerable relevance among suicidal youth. Furthermore, suicidal adolescent inpatients' self-reported affiliation with deviant peers has indirectly predicted greater suicidal ideation through increased substance use and depression (Prinstein, Boergers, Spirito, Little, 
\& Grapentine, 2000). Importantly, these authors found an independent, direct pathway between support from close friendships and less suicidal ideation suggesting that healthy and detrimental peer influences can be disentangled. Whether peer support explains variation in suicidal adolescents' psychopathology beyond what is accounted for by other sources, and whether contributions are positive or negative needs to be considered.

\section{Gender- and Age-Related Variations in Adolescents' Social Support}

In non-clinical populations, the type and amount of support individuals receive and perceive differs by gender (Piko, 1998; Vaux, 1985). For example, adolescent girls generally perceive stronger relationships with peers and family than do boys (Slavin, 1991). Furthermore, gender differences also are evident in terms of the relevance of social support to adolescents' psychological well-being and impairment (Piko, 1998; Vaux, 1985). Specifically, Slavin and Rainer (1990) found that high school girls perceived greater support from non-family adults and peers than did boys, and that girls' perceptions were more strongly associated with depressive symptoms. Additionally, support from these sources was associated with reductions in depressive symptoms over time for girls but not boys. Similarly, Mazza and Reynolds (1998) reported that, in a community sample of adolescents, higher social support predicted lower levels of suicidal ideation one year later among females only. Thus, adolescent gender appears to be an important moderator of social support effects in normative populations. Few studies of social support and psychopathology in clinical populations have considered gender effects, though diagnoses, comorbidity, and suicide-related behavior (e.g. suicidal ideation, attempt lethality) are differentially patterned for male and female adolescents (Gould, Greenberg, Velting \& Shaffer, 2003; Kandel, Raveis, \& Davies, 1991; LanghinrichsenRohling, Lewinsohn, \& Seeley, 1998).

Studies of social support in clinical populations also must place findings in a developmental context. Research with non-clinical, adolescent populations suggests that, with age, attachment to and perceived social support from family members decreases while reliance on peers for support increases (Buist, Dekovic, Meeus, \& Van Aken, 2002; Steinberg \& Silverberg, 1986). Thus, in studies conducted within clinical populations, observed declines in the strength of adolescents' connections with family may reflect normative decreases in dependence on parents, and not necessarily dysfunctional family processes; likewise, increases in support from non-family adults and peers are normative and may not indicate overreliance on these sources to the exclusion of healthy family relationships (Steinberg \& Silverberg, 1986; Vaux, 1985). Thus, associations among suicidal adolescents' social support, gender, and age require further study, and must be interpreted with reference to normative findings.

\section{The Present Study}

In the present study, the relevance of multiple sources of social support to psychiatric impairment is examined in a group of hospitalized, suicidal adolescents. Social support, as measured in the present study, refers to frequent, close, satisfying personal contact. As reviewed in Williams, Barclay, and Schmied (2004), this meaning is consistent with the emotional dimensions of social support (e.g. feeling positively regarded, validated, and able to confide), the perceptions of which are often studied relative to mental health. The broad construct of social support also can refer to the provision of pragmatic and informational resources that are not measured in the current study (Williams et al., 2004). Whether suicidal adolescents' perceptions of social support from different sources vary with age and differ by gender in ways that parallel normative trends has not been established. Thus, we hypothesize that older age will be linked with perceptions of increased levels of peer and non-family adult support and decreased family support among suicidal adolescents, as has been observed in normative community samples (Buist et al., 2002; Slavin, 1991; Vaux, 1985). Similarly, it also is hypothesized that boys will report lower perceived support across relationship types (Piko, 1998; Slavin, 1991).

Additionally, perceptions of support from multiple sources (family, non-family adults, and peers) may or may not be differentially and/or independently related to psychopathology in suicidal adolescents. Few studies have examined whether non-family sources overlap with, supplement, or interact with family support to predict psychopathology, and none have done so among suicidal adolescents. Additionally, how social support relates to impairment is hypothesized to depend on type of psychopathology. Previous research suggests that low support is associated with greater severity of adolescents' depressive symptoms and suicidal ideation (e.g. Barrera \& Garrison-Jones, 1992; Mazza \& Reynolds, 1998). However, relations between perceived support and other dimensions of these adolescents' psychopathology need to be examined. Specifically, substance abuse and externalizing behaviors are commonly associated with adolescent suicidality (e.g. Brent et al., 1994; Shafii, Carrigan, Whittinghill, \& Derrick, 1985), and both types 
of psychopathology have been linked to family and peer support in other populations (Prinstein et al., 2001; Wills $\&$ Cleary, 1996). Thus, consistent with previous work, low family support is hypothesized to be associated with increased depression, suicidal ideation, substance abuse, and externalizing behaviors. It also is expected that lower support from non-family adults will be related to greater levels of psychopathology among suicidal adolescents. Higher levels of perceived peer support are expected to be related to lower levels of suicidal ideation and depression (Prinstein et al., 2000), but may be associated with increased substance abuse and externalizing behaviors. Also, whether different sources of social support interact statistically to explain variations in psychopathology (e.g. peer support in cases of low family support) will be examined in an exploratory fashion.

Finally, consistent with other findings on the greater salience of social support for girls' functioning (reviewed in Vaux, 1985), associations between low perceived social support and psychopathology are hypothesized to be stronger for girls than for boys across social relationship types and symptom measures. Findings from the present study will inform efforts to utilize social support, a potentially powerful resource, in the treatment of suicidal adolescents.

\section{METHOD}

\section{Participants}

Participants were 220 adolescents (152 females, 68 males), ages 12 to 18 years ( $M=15.3$ years; $S D=1.5$ years) who had been psychiatrically hospitalized. Participants were recruited from two large, acute care units in the Midwestern United States, one university-based and one private hospital; mean length of stays during the two study years were 7.1 and 6.5 days. All participants had made a suicide attempt or had expressed suicidal intent, or significant suicidal ideation, and obtained a score of 20 or 30 on the Self-Harm Subscale of the Child and Adolescent Functional Assessment Scale (Hodges \& Wong, 1996). A score of 20 reflects a major or persistent disruption in functioning and, if based on suicidal ideation, requires that the youth "talks or repeatedly thinks about harming self, killing self, or wanting to die." A score of 30 indicates clear suicidal intent or a suicidal attempt. There were no diagnostic inclusion criteria. Exclusion criteria were: severe or profound mental retardation (defined by special education certification) or incapacitating psychosis (i.e. clinical ascertainment of highly impaired reality testing with an inability to complete self-report questionnaires or participate in interviews).
The racial/ethnic composition of the sample was 83\% Caucasian, 9\% African-American, 3\% biracial, 2\% Asian-American/Pacific Islander, 1\% Hispanic, $1 \%$ Native American, and 1\% "Other" or not identified. This proportion of Caucasian participants is consistent with state census data (83.4\%; U.S. Census Bureau, 2000). Participants lived with: both biological or adoptive parents (47\%), their parent and step-parent (18\%), single mother $(22 \%)$, single father $(2 \%)$, mother and other adult $(8 \%)$, or one or more non-parent caregivers (2\%). No participants lived in foster care or without an adult caregiver. A range of socioeconomic status was represented. The mean socioeconomic index scores (Stevens \& Featherman, $1981)$ for mothers and fathers were $43.0(S D=14.8)$ and $43.0(S D=21.8)$ (e.g. technicians and semiprofessionals), respectively; scores ranged from 12.67 (e.g. unskilled laborers) to 87.14 (e.g. professionals). Adolescents' annual family incomes ranged from below $\$ 10,000$ to over $\$ 250,000$, with $7 \%$ below $\$ 20,000$ and $15 \%$ above $\$ 100,000$; median family income was $\$ 40,000$ to $\$ 49,999$.

\section{Measures}

The Perceived Emotional/Personal Support Scale. (PEPSS; Slavin, 1991) assesses perceived emotional support. Respondents are instructed to list the gender and first initial of three important people in each of three relationship categories: family members, non-family adults, and friends/coworkers. Using a four point scale (hardly at all to very much), respondents answer the following questions about each person listed: "How much do you talk to them about personal concerns?," "How close do you feel to them?," and "How satisfied are you with the help and support they give you?" A fourth question, "How much do they talk to you about their concerns?," was deemed inappropriate to the assessment of social support from family members and non-family adults (e.g. grandmothers, therapists, teachers), and thus was only included in the measure of peer support. Three support variables were created by averaging all ratings for all persons listed within each relationship category: perceived support from family, non-family adults, and peers. Thus, scores ranged from 1 to 4. Slavin's (1991) factor analysis suggests that perceptions of social support are consistent within each of these categories and are relatively independent of those for the other two categories. Cronbach's alphas for family, non-family adult, and peer support scales for the current sample were $.87, .88$, and .93 , respectively.

The Beck Hopelessness Scale. (BHS; Beck \& Steer, 1988), a 20-item true/false scale, was used to measure 
participants' negative attitudes about the future. Scores range from 0 to 20 , with higher scores indicating greater hopelessness. The BHS has been shown to predict eventual suicide in adult psychiatric inpatients and outpatients (Beck \& Steer, 1988), and when used with psychiatric inpatient adolescents has a factor structure similar to that reported for adults (Steer \& Beck, 1993). The BHS is frequently used as a measure of hopelessness in studies of adolescent depression and suicidal behavior (e.g. Goldston, Daniel, \& Reboussin, 1999).

The Reynolds Adolescent Depression Scale. (RADS; Reynolds, 1987) is a 30-item self-report questionnaire that assesses presence and severity of depressive symptoms. Adolescents rate the severity of recent depressive symptoms on a 4-point Likert scale ranging from almost never to most of the time. Examples include "I feel sad" and "I feel that no one likes me." Scores range from 30 to 120, with higher scores indicating higher levels of depressive symptoms. The RADS has been shown to have strong test-retest reliability (.80), internal consistency (.92), and concurrent validity (Reynolds, 1987).

The Suicidal Ideation Questionnaire-Junior. (SIQJR; Reynolds, 1988) is a 15-item self-report questionnaire that assesses the frequency of a range of suicidal thoughts. Adolescents rate the frequency of suicidal thoughts (e.g. "I thought about killing myself" and "I wished that I had never been born") on a 7-point scale ranging from I never had this thought to almost every day. Scores range from 0 to 90 , with higher scores indicating more severe/frequent suicidal ideation. The SIQ-JR has strong, documented psychometric properties (Reynolds, 1988, 1992), and predicts suicidal thoughts and attempts 6 months following hospitalization (King et al., 1997).

The Personal Experience Screening Questionnaire. (PESQ; Winters, 1991) was used to assess participants' alcohol and substance abuse. The problem scale consists of 18 questions about the frequency with which respondents have engaged in behaviors indicative of alcohol/drug abuse. Respondents answer each question with one of four frequency ratings (never to often). Problem scale scores range from 18 to 72 ; higher scores indicate a more severe problem. Fifty participants (22\%) who exceeded cut-off scores on the Defensiveness or Infrequency scales were excluded from analyses using PESQ data.

The Externalizing Subscale of the Youth Self-Report for Ages 11-18. (YSR; Achenbach, 1991) was used to assess severity of delinquency and aggression, based on frequency ratings ("not true," "somewhat/sometimes true," or "very/often true") for a range of behavior problems (e.g. "I argue a lot"). Total scores range from 0 to 60 . The YSR is widely used in adolescent research due to its strong psychometric properties (Achenbach,
1991). The scale was developed on a sample of 1,272 clinically-referred youth, and was normed on 1,315 nonreferred youth.

In lieu of clinical diagnoses, we categorized participants with and without clinically significant levels of depression and conduct problems based on clinical cut-off scores for the RADS (total score $\geq 77$ ) and YSR Externalizing subscale (T-score $\geq 70$ ), respectively.

The Spectrum of Suicide Behavior Scale (SSB; Pfeffer, 1986) is a 5-category clinician-rated, coding system used to assess suicidality (none, suicidal ideation only, suicidal intent/threat, suicidal gesture/mild attempt, serious suicide attempt). In the present study, the SSB was used to assess history of suicide attempt(s) versus suicidal ideation only. Codes were based on all available data including parent and youth report and hospital records. The SSB has high inter-rater reliability (King et al., 1993), and has been found to predict suicidal ideation six months after hospital discharge (King et al., 1997).

\section{Procedures}

The sample was drawn from a larger, IRB-approved study of a social support intervention for psychiatrically hospitalized adolescents. Adolescent admissions to participating hospitals were screened. If information indicated that the adolescent met study criteria, the parent/guardian and adolescent were approached for informed consent and assent, respectively, within $72 \mathrm{hr}$ of admission. We were able to recruit $35 \%$ of those adolescent patients who were found to meet eligibility criteria (54\% at university hospital, $28 \%$ at private psychiatric hospital). The approximately one hour assessment was conducted during participants' psychiatric hospitalization, on average 0.8 days $(S D=2.1)$ following youth study assent.

\section{RESULTS}

\section{Participant Characteristics}

According to the SSB, $67 \%$ of participants had made at least one suicide attempt in their life, and $26 \%$ exceeded the clinical cut-off on the YSR Externalizing scale. A greater proportion of females $(60 \%)$ than males $(46 \%)$ exceeded the cut-off on the RADS [ $\left.\chi^{2}(1)=3.9, p=.049\right]$. Descriptive statistics for measures of psychopathology and social support are reported in Table I. Means reflect the severity of the psychopathology among study participants. Measures of hopelessness, depression, and suicidal ideation (BHS, RADS, and SIQ-JR) intercorrelated with 
Table I. Descriptives Statistics for Measures of Psychopathology and Perceptions of Social Support.

\begin{tabular}{lccc}
\hline & Total & Males & Females \\
& $M(S D)$ & $M(S D)$ & $M(S D)$ \\
\hline Psychopathology & & & \\
BHS $(N=218)$ & $8.7(6.1)$ & $7.6(5.7)$ & $9.2(6.2)$ \\
RADS $(N=220)$ & $78.5(17.0)$ & $73.8(17.6)$ & $80.6(16.4)$ \\
SIQ-JR $(N=217)$ & $42.0(22.8)$ & $39.1(22.3)$ & $43.3(23.0)$ \\
PESQ $(N=170)$ & $26.7(11.2)$ & $25.1(10.8)$ & $27.5(11.4)$ \\
YSR-Ext $(N=216)$ & $21.0(9.2)$ & $21.2(10.0)$ & $20.9(8.9)$ \\
Social Support & & & \\
Family $(N=220)$ & $2.82(0.68)$ & $2.96(0.69)$ & $2.76(0.67)$ \\
Non-Family Adults & $2.73(0.71)$ & $2.69(0.79)$ & $2.75(0.68)$ \\
$\quad(N=201)$ & & & \\
Peers $(N=217)$ & $3.28(0.59)$ & $3.11(0.72)$ & $3.36(0.51)$ \\
\hline
\end{tabular}

Note. BHS: Beck Hopelessness Scale; RADS: Reynolds Adolescent Depression Scale; SIQ-JR: Suicidal Ideation Questionnaire, Adolescent version; PESQ: Personal Experiences Screening Questionnaire (Alcohol/Substance Abuse); YSR-Ext: Externalizing Behavior Problem subscale of Youth Self-Report. Higher scores on measures of psycholopathology indicate greater levels of dysfunction. Higher scores on social support measures suggest greater levels of perceived support.

moderate strength $(r=.56$ to .71 , all $p<.001)$, as did measures of alcohol/substance abuse and externalizing behavior problems (PESQ and the Externalizing scale of the YSR; $r=.40, p<.001)$; measures across these domains were modestly correlated $(r=.19$ to .32 , all $p<.01)$.

Correlations among levels of social support from the three relationship types also were examined. Among female participants, levels of perceived social support from family, non-family adult, and peer sources were positively associated [ $r^{6}$ s $(143)$ ranged from .32 to $\left..48, p<.001\right]$. Among male participants, levels of family and non-family adult support were positively related to each other $[r(58)$ $=.43, p<.01]$, but neither related to support from peers.

\section{Age and Gender Effects for Psychopathology and Social Support Measures}

Age and gender effects on psychopathology were examined using linear regression analyses. In separate models by outcome, adolescent gender, age, and the gender by age interaction were considered as independent predictors of the five measures of psychopathology and the three sources of perceived social support. The regression model was significant for $\operatorname{RADS}[F(3,219)=2.7, p$ $=.045]$; gender was the only significant predictor $(\beta=$ $-.19, p=.006)$, suggesting that girls had higher levels of depressive symptoms. Models for PESQ $[F(3,169)=$ $4.6, p=.004]$ and YSR Externalizing $[F(3,215)=2.9$, $p=.034]$ also were significant. Each revealed a significant effect for age only; older age was associated with higher alcohol/substance use problem scores $(\beta=.19, p=.013)$ and fewer externalizing symptoms $(\beta=-.20, p=.004)$.

In models predicting perceived social support, only the model for peer support was significant $[F(3,216)=$ $5.1, p=.002]$. There was a significant effect for gender $(\beta=-.18, p=.008)$, and a trend for age $(\beta=.12, p$ $=.071)$, that reached significance when the interaction was omitted from the model $(\beta=.15, p=.031)$. Females perceived higher levels of emotional support from peers; age was positively associated with peer support. The model predicting peer support was re-run, controlling for whether participants were above or below the clinical cut-offs on RADS and YSR Externalizing, and for suicide attempt status. In these models, beta values for gender and age effects on peer support did not change, and remained significant, suggesting that type of psychopathology and attempt history did not account for observed gender effects.

\section{Relationships Between Perceived Social Support and Psychopathology}

Pearson correlations between measures of social support and psychopathology are presented separately for females and males in Table II. Support ratings were negatively associated with hopelessness (BHS) and depression severity (RADS) among females, but not males. Poorer

Table II. Gender-Specific Correlations Among Measures of Psychopathology and Perceptions of Social Support.

\begin{tabular}{lccccc}
\hline Support source & BHS & RADS & SIQ-JR & PESQ & $\begin{array}{c}\text { YSR- } \\
\text { Ext }\end{array}$ \\
\hline Female Participants & & & & & \\
$\quad$ Family & $-.43^{* * *}$ & $-.36^{* * *}$ & $-.28^{* *}$ & -.11 & -.16 \\
Adult & $-.21^{*}$ & $-.24^{* *}$ & -.13 & .08 & -.12 \\
$\quad$ Peer & $-.18^{*}$ & $-.18^{*}$ & -.12 & .11 & .06 \\
Male Participants & & & & & \\
$\quad$ Family & -.11 & -.02 & -.04 & $-.39^{* *}$ & -.09 \\
Adult & -.06 & .16 & .05 & -.15 & .11 \\
Peer & .23 & .24 & $.30^{*}$ & .15 & .12 \\
\hline
\end{tabular}

Note. $N$ `s for specific correlations ranged from 105-152 for girls and 48-68 for boys.

BHS: Beck Hopelessness Scale; RADS: Reynolds Adolescent Depression Scale; SIQ-JR: Suicidal Ideation Questionnaire, Adolescent version; PESQ: Personal Experiences Screening Questionnaire (Alcohol/Substance Abuse); YSR-Ext: Externalizing Behavior Problem subscale of Youth Self-Report. Higher scores on measures of psycholopathology indicate greater levels of dysfunction. Higher scores on social support measures suggest greater levels of perceived support. ${ }^{*} p<.05 .^{* *} p<.01 .^{* * *} p<.001$ 


\section{Suicidal Adolescents' Social Support}

family support was associated with more severe suicidal ideation among females, and alcohol/substance abuse problem scores among males. There also was evidence that males' perceptions of greater peer support were related to higher levels of suicidal ideation.

Whether social support from multiple sources related to measures of psychopathology, and whether observed effects were moderated by gender was examined using regression analyses. In separate models, each measure of psychopathology was examined as the dependent variable; age was controlled in YSR Externalizing and PESQ models. Due to concerns over statistical power, all three sources of support in interaction with gender were not examined simultaneously. Instead, since poor family support has been most consistently associated with psychopathology in past research, we considered whether family support and one of the other two sources of support were independently associated with variation in severity of psychopathology. Independent variables were centered prior to entry into models. Significant interactions were probed by calculating simple slopes for the support measures for females and males (Aiken \& West, 1991).

In the first set of models, participant gender, family and peer support, and the interactions of each source of support with gender were considered as independent variables. All models were significant (see Table III). In Model 1, family support alone and in interaction with gender were significant predictors of BHS. Post hoc probing suggested that lower levels of family support were associated with higher levels of hopelessness among females $(\beta=-.42, p<.001)$, but not males $(\beta=-.08, p$ $=$ n.s.). In Model 2 , family support and the interactions of gender with support from both family members and peers predicted RADS scores. Post hoc testing revealed that greater depression severity was predicted by lower family support for females $(\beta=-.32, p<.001)$, but not for males ( $\beta=-.00, p=$ n.s. $)$, and higher peer support for males $(\beta=.20, p=.039)$, but not females $(\beta=-.08, p=$ n.s. $)$. In Model 3, SIQ-JR scores were predicted by perceptions of family support and by the interaction of gender and peer support; there was a trend for the interaction between gender and family support. Probing of simple slopes suggested that more severe suicidal ideation was associated with perceptions of lower family support among females $(\beta=-.26, p=.002)$, but not males $(\beta=.00, p=$ n.s. $)$, and higher peer support among males ( $\beta=.24, p=.016)$, but not females $(\beta=-.04, p=$ n.s. $)$. In Model 4 , participant age and perceived support from family and peer sources each were independent predictors of YSR Externalizing problems. Specifically, younger age $(\beta=-.24, p<.001)$ and perceptions of poorer family support $(\beta=-.18, p=$
Table III. Summary of Regression Models for Variables Predicting Adolescents' Psychopathology

\begin{tabular}{|c|c|c|c|}
\hline Variable & $\mathrm{B}$ & SE (B) & $\beta$ \\
\hline \multicolumn{4}{|l|}{ Model 1: Hopelessness (BHS) } \\
\hline Gender & -1.00 & 0.86 & -.08 \\
\hline Family support & -1.92 & 0.41 & $-.32^{* * *}$ \\
\hline Peer support & 0.12 & 0.43 & .02 \\
\hline Gender $\times$ family support & 2.09 & 0.86 & $.16^{*}$ \\
\hline Gender $\times$ peer support & 1.29 & 0.82 & .11 \\
\hline \multicolumn{4}{|c|}{ Model 2: depressive symptoms (RADS) } \\
\hline Gender & -5.77 & 2.43 & $-.16^{*}$ \\
\hline Family support & -3.82 & 1.15 & $-.22^{* *}$ \\
\hline Peer support & 0.12 & 1.22 & .01 \\
\hline Gender $\times$ family support & 5.43 & 2.43 & $.15^{*}$ \\
\hline Gender $\times$ peer support & 4.78 & 2.30 & $.15^{*}$ \\
\hline \multicolumn{4}{|c|}{ Model 3: suicidal ideation (SIQ-JR) } \\
\hline Gender & -2.06 & 3.37 & -.04 \\
\hline family support & -4.10 & 1.59 & $-.18^{*}$ \\
\hline peer support & 1.02 & 1.70 & .05 \\
\hline Gender $\times$ family support & 6.05 & 3.37 & $.12^{\dagger}$ \\
\hline Gender $\times$ peer support & 6.53 & 3.19 & $.15^{*}$ \\
\hline \multicolumn{4}{|c|}{ Model 4: externalizing symptoms (YSR-Ext) } \\
\hline Age & -1.54 & 0.44 & $-.24^{* * *}$ \\
\hline Gender & 0.85 & 1.39 & .04 \\
\hline Family support & -1.71 & 0.67 & $-.18^{*}$ \\
\hline Peer support & 1.48 & 0.70 & $.16^{*}$ \\
\hline Gender $\times$ family support & 0.79 & 1.41 & .04 \\
\hline Gender $\times$ peer support & -0.11 & 1.31 & -.01 \\
\hline \multicolumn{4}{|c|}{ Model 5: alcohol/substance abuse (PESQ) } \\
\hline Age & 1.45 & 0.58 & $.20^{*}$ \\
\hline Gender & -1.00 & 1.82 & -.04 \\
\hline Family support & -2.06 & 0.89 & $-.18^{*}$ \\
\hline Peer support & 1.50 & 0.95 & .13 \\
\hline Gender $\times$ family support & -1.75 & 1.85 & -.07 \\
\hline Gender $\times$ peer support & -1.82 & 1.76 & -.09 \\
\hline
\end{tabular}

Note. Model 1 (BHS) $F(5,214)=8.0, p<.001$; Adj. $R^{2}=.14$; Model $2(\mathrm{RADS}) F(5,216)=6.8, p<.001$; Adj. $R^{2}=.12$; Model 3 (SIQ-JR) $F(5,214)=3.9, p=.002$; Adj. $R^{2}=.06$; Model 4 (YSRExt) $F(6,212)=3.2, p=.005$; Adj. $R^{2}=.06$; Model 5 (PESQ) $F(6$, 166) $=3.3, p=.004$; Adj. $R^{2}=.08$

${ }^{\dagger} p<.10 .^{*} p<.05 .^{* *} p<.01 .^{* * *} p<.001$.

$.011)$ and greater peer support $(\beta=.16, p=.035)$ were uniquely associated with externalizing problems. Finally, in Model 5, PESQ problem scores were significantly predicted by participant age $(\beta=.20, p=.013)$ and family support $(\beta=-.18, p=.022)$; older age and lower levels of family support were each related to higher problem scores.

The second set of models paralleled the first, except that non-family adult support and its interaction with gender were examined in place of peer support. Significant findings involving family support were essentially unchanged. However, non-family adult support did not emerge as an independent predictor of psychopathology alone or in interaction with gender. 
Although all participants required psychiatric hospitalization for severe psychopathology, previous analyses showed that severity of depressive symptoms varied by gender. Thus, we questioned whether the observed moderating effects of gender were an artifact of clinical features of youth that covaried with gender. We examined this issue by re-running those models that had yielded significant gender by support interactions, replacing gender with one of the following dichotomous clinical grouping variables: clinical threshold on RADS or YSR Externalizing scale, or attempt history. For example, we attempted to predict SIQ-JR scores from age, attempt history, family and peer support, and the interactions of attempt history with family and peer support. None of these models yielded significant interaction terms. Thus, findings did not support the alternative hypothesis that clinical presentation moderates associations between perceived social support and psychopathology.

A final set of exploratory regressions showed that family support did not interact with peer or non-family adult support to predict any of the measures of psychopathology.

\section{DISCUSSION}

In the current study, the social support perceived by a large sample of suicidal adolescents was examined in relation to their psychopathology in greater detail than has been reported previously. Measurement of suicidal ideation, internalizing and externalizing symptoms, and alcohol/substance abuse made it possible to relate perceptions of social support to the multiple dimensions of psychopathology with which suicidal adolescents present. That the study considered a breadth of sources from which adolescents draw social support is another strength of the present study. Additionally, since participants completed assessments within days of a psychiatric hospitalization, this study provides a view of these adolescents' support and impairment at a critical point in their lives.

A central finding was that female adolescents' perceptions of low family support were related to greater levels of hopelessness, depressive symptoms, and suicidal ideation. These findings are consistent with other research documenting the connection between low family support and suicidal ideation, suicide attempts and depression in community and outpatient adolescent samples (e.g. Cumsille \& Epstein, 1994; Perkins \& Hartless, 2002) and to subsequent suicidal ideation and behavior in depressed and hospitalized adolescents (e.g. King et al., 1995). Additionally, in general, adolescents' perceptions of low family support were associated with greater externalizing behav- ior problems and alcohol and substance abuse. Relationships between support and these latter two dimensions of psychopathology have not been reported previously in a sample of suicidal adolescents, though these youth frequently present with these symptoms (Brent et al., 1994; Shafii et al., 1985).

Also prominent was the finding that variations in perceived family support were not related to hopelessness, depression severity, or suicidal ideation among male participants. The greater salience of social support to females' compared to males' psychological well-being, psychopathology, and suicidal behavior has been reported in other research with clinical and non-clinical populations (e.g. Lewinsohn et al., 2001; Mazza \& Reynolds, 1998; Piko, 1998; Slavin \& Rainer, 1990), an issue that will be examined in greater detail below.

Perceptions of peer support also were related to suicidal adolescents' psychopathology in gender-specific ways. Surprisingly, greater levels of perceived peer support were associated with higher levels of hopelessness, depressive symptoms, and suicidal ideation among males, independent of variations in family support. It is possible that suicidal youth affiliate with and are negatively influenced by depressed and suicidal peers. Indeed, others have reported associations between the internalized distress and suicidal behavior of adolescents and that of their friends (e.g. Hogue \& Steinberg, 1995; Leslie, Stein, \& Rotheram-Borus, 2002; Prinstein et al., 2000). Recent research suggests these associations are due to a combination of: non-causal self-selection (i.e. homophily); direct peer influence via modeling and differential reinforcement of affect; and indirect influence, whereby affiliation with antisocial peers leads to risk-taking and negative consequences that then precipitate or exacerbate depression (e.g. Fergusson, Wanner, Vitaro, Horwood, \& Swain-Campbell, 2003; Heller \& Tanaka-Matsumi, 1999). In contrast to these patterns, peer support failed to explain variation in females' psychopathology after accounting for family support. Thus, perceptions of peer support appeared to have quite different relationships to the psychopathology of suicidal male and female adolescents.

Associations between lower levels of peer support and adolescent depressive symptoms have been documented, though results have depended on the population. For example, in community samples of adolescents, less peer support predicted more symptoms of depression (Slavin \& Rainer, 1990), and, among adolescent males, predicted suicide attempts in young adulthood (Lewinsohn et al., 2001). In contrast, within a sample of adolescent outpatients, Cumsille and Epstein (1994) did not find depression and peer support to be related. Thus, 
the lack of relationship between suicidal adolescent females' peer support and psychopathology may be consistent with the trend that, in more restricted samples of troubled populations, the relevance of peer support may be overshadowed by that of family support.

Based on previous work, we considered whether greater peer support might be related to increased alcohol and substance abuse and externalizing behavior (e.g. Dishion et al., 1996). In general, greater peer support was related to more externalizing problems, but not to alcohol or substance abuse. Importantly, the current study did not distinguish between social support from prosocial and antisocial peers. Thus, it is possible that relationships between peer support and psychopathology were clouded by the aggregation of positive and negative influences. Indeed, Hartup (1996) argues that the identities of friends and dyadic qualities of friendships are more significant to the study of individual differences than is the simple presence or absence of friends or the overall level of support provided. Others have found that the contexts (e.g. in or out of school) in which adolescent friendships are formed and maintained are important (Kiesner, Kerr, $\&$ Stattin, 2004). Thus, future work should identify not only the level, but also the nature of peer support in relation to suicidal adolescents' psychopathology. Further research may uncover why suicidal adolescents' perceptions of peer relationships seem to be so disconnected from their functioning. For example, their peer contacts may be perceived as intensely fulfilling, but may be conflictual, unstable, and short-lived, and may be with other psychiatrically impaired youth.

Perceived support from non-family adults was not related to adolescent psychopathology after controlling for family support. It is possible that the weak supportive networks of suicidal adolescents might explain this finding. That is, previous reports suggest that, compared to other youth, suicidal adolescents are less integrated into school, have less positive experiences with school and teachers, and are less involved in community and extracurricular activities (e.g. Kandel et al., 1991; Mazza \& Eggert, 2001; Perkins \& Hartless, 2002). Further research is needed to determine whether enhancing support from non-family adults can be an important part of suicide prevention and intervention efforts (e.g. King et al., in press).

Next, previous research suggested that peer and nonfamily adult support might be important in combination with certain levels of family support (e.g. Barrera \& Garrison-Jones, 1992; Cumsille \& Epstein, 1994; Gauze et al., 1996). For example, one might expect that adolescents with low levels of family support might experience even more profound impairment if they also had low non-family adult support. In the current study, how- ever, exploratory analyses did not suggest that associations between psychopathology and support from a particular source depend on the levels of support from others sources. Future research should examine whether variations in peer and non-family adults support alone or in interaction with family support may predict improved functioning longitudinally, as reported by Slavin and Rainer (1990).

Age-related trends in suicidal adolescents' perceived social support were partially consistent with normative findings. Higher peer support was associated with increased age, mirroring normative trends toward increased affiliation and reliance on peers across adolescence (e.g. Steinberg \& Silverberg, 1986). However, age was not associated with levels of perceived family support. In contrast, others have observed such an age effect (Slavin, 1991), which may reflect normative age-related processes, such as greater negative perceptions of the family and decreased parental influence on identity development (Garnefski, 2000; Meeus \& Dekovic, 1995). Further research may uncover why this normative pattern of increased distancing from family was not evident among suicidal adolescents.

The present results are relevant to research on gender differences in social support in clinical and non-clinical populations. Suicidal male and female adolescent participants differed on levels of perceived peer support and on the links that family and peer support had with psychopathology. Notably, gender differences in symptom severity and suicide attempt history did not account for these gender differences. Regarding the potentially detrimental power of peer support, others have found that male adolescents are more susceptible to peer influence, both positive and negative (e.g. Hogue \& Steinberg, 1995). With respect to the positive nature of family support, the gender differences observed in the current study and in previous work may reflect that support measures generally emphasize the emotional dimensions of social support, which, for reasons that are not clear, may be less relevant to males. Others have found that males appear to receive and/or perceive lower levels of social support, and that males' experience of emotional support appears to be less related to psychopathology and psychological well-being (Mazza \& Reynolds, 1998; Piko, 1998; Slavin \& Rainer, 1990; Vaux, 1985). Levels of other types of support (e.g. rational-material, informational, practical) also appear to differ by gender and are differentially related to boys' and girls' psychological distress and well-being (Piko, 1998; Valery, O'Connor, \& Jennings, 1997). Future research should examine how psychopathology relates to the presence of these other features of social support that were not measured in the present study (reviewed in Williams 
et al., 2004) but that may be more typical of boys' relationships with friends, family, and adult mentors. Further work also may clarify whether gender differences in social support are explained by differences in tendencies to seek out support, skills at identifying and/or engaging supportive persons, ability to benefit from support, or artifacts of self-report (e.g. Vaux, 1985). Better understanding of these issues is essential, given recent proposals to mobilize social support in adolescent suicide prevention and intervention programs, and recent findings that the effectiveness of such efforts may depend on adolescent gender (King et al., in press).

\section{Study Limitations}

There were several limitations to the current study. First, the study was cross-sectional, limiting conclusions that could be drawn regarding the causal direction of associations between support and psychopathology. Second, though the measures of psychopathology used in the present study have strong, documented psychometric properties in clinical populations, another limitation was the reliance on self-report measures. Measures of perceived social support may be vulnerable to bias among individuals with severe depression, since depressive cognitions may distort evaluation of theoretically distinct constructs (e.g. Brendgen, Vitaro, Turgeon, \& Poulin, 2002). For example, Slavin and Compas (1989) reported inadequate discriminant validity between depressive symptoms and subjective appraisals of social support. In contrast, Barrera, Baca, Christiansen, and Stohl (1985) have shown that outpatients' self-reports of their social support networks can be externally corroborated. Also, Cutrona (1989) has demonstrated that studies of social support that control for depression may underestimate the importance of this construct by attributing all shared variance to depression. Indeed, "distortions" may have a significant impact on later functioning, beyond what is explained by the objective features of interpersonal relationships (reviewed in Brendgen et al., 2002). As in the current study, others have also found that perceptions of high social support sometimes are associated with greater impairment, such as greater depression severity (Barrera \& Garrison-Jones, 1992) and depression relapse (Veiel, 1993), findings that are not easily dismissed as shared variance artifacts.

Third, although the current study provides rich information on the social support and psychopathology of a large group of adolescents in crisis, this strength also may limit generalizability. That is, psychiatrically hospitalized adolescents may experience a swell of parental support or, alternatively, increased family disorganization, resent- ment, and/or withdrawal of support that may or may not have long-term stability or relevance once the suicidal crisis ends. Additionally, clinicians may use their assessment of the adequacy of family support to judge whether psychiatric hospitalization is appropriate (Dicker et al., 1997), particularly in adolescents for whom the level of imminent suicide risk is difficult to ascertain. For this reason, it is possible that relationships between family support and psychopathology are inflated relative to the broader population of suicidal adolescents. On the other hand, it is likely that some patterns may not have been significant due to decreased variability in measures of psychopathology among this highly impaired sample. Additionally, the smaller number of males in the sample may have limited the statistical power needed to detect some effects.

Fourth, our relatively low recruitment rate $(35 \%)$ may limit generalizability. Although the gender (Borst \& Noam, 1989) and racial distributions were similar to those of other studies and to state census data, it is unknown whether participating and refusing youth differed in other important ways, such as on severity of psychopathology or extent of social support network. It is difficult to predict how youth who did or did not participate would differ on these dimensions, and how potential differences would impact the present results. It is possible, for example, that youth suffering profound hopelessness and social isolation would refuse to participate, thus contributing to an underestimate of the link between hopelessness and social support.

Finally, youth with elevated "lie scale" scores on the measure of alcohol/substance abuse (PESQ) were excluded from analyses using this measure. Findings based on the PESQ and other measures of psychopathology were similar in strength and direction. Nevertheless, findings may not generalize to youth who are motivated to exaggerate or minimize their alcohol/substance abuse (e.g. to appear adventurous or due to denial about substance dependence).

\section{CONCLUSIONS}

The present study underscores that associations between perceptions of social support and psychopathology among suicidal adolescents are complex, and depend on youth (gender, age, type of psychopathology) and social network characteristics (relationship to youth). Findings support the notion that limitations in suicidal adolescents' social support networks have important links to their psychopathology. Associations between suicidal female participants' low family support and both internalizing symptoms and suicidal ideation suggest that treatment 


\section{Suicidal Adolescents' Social Support}

efforts that address interpersonal issues, with a particular focus on family relationships, may be beneficial. However, results raise questions about the associations between social support and impairment among male, suicidal adolescents. Specifically, among males, family support appeared to be largely disconnected from psychiatric impairment, and strong peer support seemed to be associated with greater psychopathology. Longitudinal research is needed to examine the role that social support from family and non-family plays in the lives of male and female, suicidal adolescents following hospitalization. Finally, although the current sample was atypical in terms of level of impairment, gender- and age-related variations in peer social support paralleled normative findings. Taken together, findings indicate that clinicians and researchers must attend to how pathological and normal developmental processes interact, in order to understand the role of social support in the lives of adolescents hospitalized for suicidality.

\section{ACKNOWLEDGMENTS}

This research was supported by Ronald McDonald House Charities and a Student Award Program grant to the first author from the Blue Cross and Blue Shield of Michigan Foundation. The authors wish to thank Sanjeev Venkataraman, MD, for administrative support; Anne Kramer, MSW, ACSW, for project coordination; Jason Washburn, Ph.D., Carrie Hatcher-Kay, Ph.D., Neshe Sarkozy, and Jordana Ostro for data assistance. We also thank participating adolescents and their families, independent evaluators, and the inpatient staff at participating psychiatric hospitals.

\section{REFERENCES}

Achenbach, T. M. (1991). Manual for the Youth Self-Report and 1991 Profile. Burlington, VT: University of Vermont, Department of Psychiatry.

Aiken, L. S., \& West, S. G. (1991). Multiple regression: Testing and interpreting interactions. Newbury Park, CA: Sage.

Anderson, R. N. (2002). Deaths: Leading causes for 2000. National Vital Statistics Reports, 50(16), Hyattsville, MD: National Center for Health Statistics.

Barrera, M., \& Garrison-Jones, C. (1992). Family and peer social support as specific correlates of adolescent depressive symptoms. Journal of Abnormal Child Psychology, 20, 1-16.

Beck, A. T., \& Steer, R. A. (1988). Beck Hopelessness Scale Manual. New York: Harcourt Brace Jovanovich.

Borst, S. R., \& Noam, G. G. (1989). Suicidality and Psychopathology in hospitalized children and adolescents. Acta Paedopsychiatry, 52, $165-175$.
Buist, K., Dekovic, M., Meeus, W., \& Van Aken, M. (2002). Developmental patterns in adolescent attachment to mother, father, and sibling. Journal of Youth and Adolescence, 31, 167-176.

Brendgen, M., Vitaro, F., Turgeon, L., \& Poulin, F. (2002). Assessing aggressive and depressed children's social relations with classmates and friends: A matter of perspective. Journal of Abnormal Child Psychology, 30, 609-624.

Brent, D. A., Johnson, B. A., Perper, J., Connolly, J., Bridge, J., Bartle, S., \& Rather, C. (1994). Personality disorder, personality traits, impulsive violence, and completed suicide in adolescents. Journal of the American Academy of Child and Adolescent Psychiatry, 33, 1080-1086.

Cumsille, P. E., \& Epstein, N. (1994). Family cohesion, family adaptability, social support, and adolescent depressive symptoms in outpatient clinic families. Journal of Family Psychology, 8, 202214

Cutrona, C. (1989). Ratings of social support by adolescents and adult informants degree of correspondence and prediction of depressive symptoms. Journal of Personality and Social Psychology, 57, 723730 .

de Wilde, E. J., Kienhorst, I. C., Diekstra, R. F., \& Wolters, W. H. (1992). The relationship between adolescent suicidal behavior and life events in childhood and adolescence. American Journal of Psychiatry, 149, 45-51.

Dicker, R., Morrissey, R. F., Abikoff, H., Alvir, J. M. J., Weissman, K., Grover, J., \& Koplewicz, H. S. (1997). Hospitalizing the suicidal adolescent: Decision-making criteria of psychiatric residents. Journal of the American Academy of Child and Adolescent Psychiatry, 36. 769-776.

Dishion, T. J., \& Owen, L. D. (2002). A longitudinal analysis of friendships and substance use bi-directional influence from adolescence to adulthood. Developmental Psychology, 38, 480-491.

Dishion, T., Spracken, K., Andrews, D., \& Patterson, G. (1996). Deviancy training in male adolescent friendships. Behavior Therapy, 27, 373-390.

Fergusson, D., Wanner, B., Vitaro, F., Horwood, L. J., \& Swain-Campbell, N. (2003). Deviant peer affiliations and depression: Confounding or causation? Journal of Abnormal Child Psychology, 31, 605-618.

Garnefski, N. (2000). Age differences in depressive symptoms, antisocial behavior, and negative perceptions of family, school, and peers among adolescents. Journal of the American Academy of Child \& Adolescent Psychiatry, 39, 1175-1181.

Gauze, C., Bukowski, W. M., Aquan-Assee, J., \& Sippola, L. K. (1996). Interactions between family environment and friendship and associations with self-perceived well-being during adolescence. Child Development, 67, 2201-2216.

Goldston, D., Daniel, S., \& Reboussin, D. (1999). Suicide attempts among formerly hospitalized adolescents: A prospective naturalistic study of risk during the first 5 years after discharge. Journal of the American Academy of Child and Adolescent Psychiatry, 38, 660-671.

Gould, M. S., Greenberg, T., Velting, D. M., \& Shaffer, D. (2003). Youth suicide risk and preventive interventions: A review of the past 10 years. Journal of the American Academy of Child and Adolescent Psychiatry, 42(4), 386-405.

Harrington, R., Kerfoot, M., Dyer, E., McNiven, F., Gill, J., Harrington, V., Woodham, A., \& Byford, S. (1998). Randomized trial of a home-based family intervention of children who have deliberately poisoned themselves. Journal of the American Academy of Child and Adolescent Psychiatry, 37, 512-518.

Hartup, W. (1996). The company they keep: Friendships and their developmental significance. Child Development, 67, 1-13.

Heller, M. C., \& Tanaka-Matsumi, J. (1999). A sequential analysis of depressive behaviors within adolescent peer interactions. Journal of Psychopathology and Behavioral Assessment, 21, 249-273.

Hodges, K., \& Wong, M. M. (1996). Psychometric characteristics of a multidimensional measure to assess impairment: The Child and 
Adolescent Functional Assessment Scale. Journal of Child and Family Studies, 5, 445-467.

Hogue, A., \& Steinberg, L. (1995). Homophily of internalized distress in adolescent peer groups. Developmental Psychology, 31, 897-906.

Huey, S. J., Henggeler, S. W., Rowland, M. D., Halliday-Boykins, C. A., Cunningham, P. B., Pickrel, S. G., \& Edwards, J. (2004). Multisystemic Therapy effects on attempted suicide by youths presenting psychiatric emergencies. Journal of the American Academy of Child and Adolescent Psychiatry, 43, 183-190.

Kandel, D., Raveis, V., \& Davies, M. (1991). Suicidal Ideation in Adolescence: Depression, substance use, and other risk factors. Journal of Youth and Adolescence, 20, 289-308.

Kiesner, J., Kerr, M., \& Stattin, H. (2004). "Very Important Persons" in adolescence: Going beyond in-school, single friendships in the study of peer homophily. Journal of Adolescence, 27, 545-560.

King, C. A. (1997). Suicidal behavior in adolescence. In R. W. Maris, M. M. Silverman, \& S. S. Canetto (Eds.), Review of Suicidology (pp. 61-95). New York: Guilford.

King, C. A., Hovey, J. D., Brand, E., Wilson, R., \& Ghaziuddin, N. (1997). Prediction of Positive Outcomes for Adolescent Psychiatric Inpatients. Journal of the American Academy of Child and Adolescent Psychiatry, 36, 1434-1442.

King, C. A., Kramer, A., Preuss, L., Kerr, D. C. R., Weisse, L., \& Venkataraman, S. (in press). Youth-nominated support team for suicidal adolescents (Version 1): A randomized controlled trial. Journal of Consulting and Clinical Psychology.

King, C. A., Segal, H., Kaminski, K., Naylor, M., Ghaziuddin, N., \& Radpour, L. (1995). A prospective study of suicidal behavior following hospitalization. Suicide and Life-Threatening Behavior, 25 , 327.

King, C. A., Segal, H., Naylor, M., \& Evans, T. (1993). Family functioning and suicidal behavior in adolescent inpatients with mood disorders. Journal of the American Academy of Child and Adolescent Psychiatry, 32, 1198-1206.

Langhinrichsen-Rohling, J., Lewinsohn, P., \& Seely, J. (1998). Gender differences in the suicide-related behaviors of adolescents and young adults. Sex Roles, 39, 839-854.

Leslie, M. B., Stein, J. A., \& Rotheram-Borus, M. J. (2002). Sex-specific predictors of suicidality among runaway youth. Journal of Clinical Child and Adolescent Psychology, 31, 27-40.

Lewinsohn, P., Rohde, P., Seeley, J., \& Baldwin, C. (2001). Gender differences in suicide attempts from adolescence to young adulthood. Journal of the American Academy of Child and Adolescent Psychiatry, 40, 427-434.

Mazza, J. J., \& Eggert, L. L. (2001). Activity involvement among suicidal and nonsuicidal high-risk and typical adolescents. Suicide and LifeThreatening Behavior, 31, 265-281.

Mazza, J., \& Reynolds, W. (1998). A longitudinal investigation of depression, hopelessness, social support, and major and minor life events and their relation to suicidal ideation in adolescents. Suicide and Life-Threatening Behavior, 28, 358-374.

Meeus, W., \& Dekovic, M. (1995). Identity development, parental and peer support in adolescence: Results of a national Dutch survey. Adolescence, 30, 931-944.

Perkins, D., \& Hartless, G. (2002). An ecological risk-factor examination of suicide ideation and behavior of adolescents. Journal of Adolescent Research, 17, 3-26.

Pfeffer, C. R. (1986). The suicidal child. New York, NY: Guilford.
Piko, B. (1998). Social support and health in adolescence: A factor analytical study. British Journal of Health Psychology, 3, 333-344.

Prinstein, M., Boergers, J., \& Spirito, A. (2001). Adolescents' and their friends' health-risk behavior: Factors that alter or add to peer influence. Journal of Pediatric Psychology, 26, 287-298.

Prinstein, M., Boergers, J., Spirito, A., Little, T., \& Grapentine, W. (2000). Peer functioning, family dysfunction, and psychological symptoms in a risk factor model for adolescent inpatients' suicidalideation severity. Journal of Clinical Child Psychology, 29, 392-405.

Reynolds, W. M. (1987). Reynolds Adolescent Depression Scale Professional Manual. Odessa, Florida: Psychological Assessment Resources.

Reynolds, W. M. (1988). Suicidal Ideation Questionnaires—Junior Professional Manual. Odessa, Florida: Psychological Assessment Resources.

Shafii, M., Carrigan, S., Whittinghill, J. R., \& Derrick, A. (1985). Psychological autopsy of completed suicide in children and adolescents. American Journal of Psychiatry, 142, 1061-1064.

Slavin, L. A. (1991). Validation studies of the PEPSS, a measure of perceived emotional support for use with adolescents. Journal of Adolescent Research, 6, 316-335.

Slavin, L., \& Compas, B. (1989). The problem of confounding social support and depressive symptoms: A brief report on a college sample. American Journal of Community Psychology, 17, 57-66.

Slavin, L. A., \& Rainer, K. L. (1990). Gender differences in emotional support and depressive symptoms among adolescents: A prospective analysis. American Journal of Community Psychology, 18, 407-421.

Spirito, A., Boergers, J., \& Donaldson, D. (2000). Adolescent suicide attempters: Post-attempt course and implications for treatment. Clinical Psychology and Psychotherapy, 1-13.

Steinberg, L., \& Silverberg, S. B. (1986). The vicissitudes of autonomy in early adolescence. Child Development, 57, 841-851.

Stevens, G., \& Featherman, D. L. (1981). A revised socioeconomic index of occupational status. Social Science Research, 10, 364-396.

U.S. Census Bureau (2000). Census 2000 Briefs and Special Reports: Overview of Race and Hispanic Origin, March 2001.

Valery, J. H., O'Connor, P., \& Jennings, S. (1997). The nature and amount of support college-age adolescents request and receive from parents. Adolescence, 32, 323-338.

Vaux, A. (1985). Variations in social support associated with gender, ethnicity, and age. Journal of Social Issues, 41, 89-110.

Veiel, H. (1993). Detrimental effects of kin support networks on the course of depression. Journal of Abnormal Psychology, 102, 419429.

Williams, P., Barclay, L., \& Schmied, V. (2004). Defining social support in context: A necessary step in improving research, intervention, and practice. Qualitative Health Research, 14, 942-960.

Wills, T. A., \& Cleary, S. D. (1996). How are social support effects mediated? A test with parental support and adolescent substance use. Journal of Personality and Social Psychology, 71, 937952.

Winters, K. C. (1991). Personal Experience Screening Questionnaire Manual. Los Angeles, California: Western Psychological Services.

Wood, A., Trainor, G., Rothwell, J., Moore, A., \& Harrington, R. (2001). Randomized trial of group therapy for repeated deliberate self-harm in adolescents. Journal of the American Academy of Child and Adolescent Psychiatry, 40, 1246-1253. 